\title{
Measuring biopsychosocial risk for back pain disability in chiropractic patients using the STarT back screening tool: a cross- sectional survey
}

\author{
Yasmeen Khan $^{1 *}$ D, Dana Lawrence ${ }^{1}$, Robert Vining ${ }^{2}$ and Dustin Derby ${ }^{3}$
}

\begin{abstract}
Background: The Keele STarT Back Screening Tool (SBT), a 9-item questionnaire, screens for pain, physical functioning, fear-avoidance beliefs, catastrophizing, anxious thoughts, low mood, and bothersomeness in persons with back pain. SBT scores designate low, medium, or high risk for developing persistent disabling back pain. The primary study aim was to report the prevalence of SBT-calculated risk for back pain disability in US patients seeking chiropractic care.

Methods: The SBT questionnaire was administered to patients $\geq 18$ years in 3 Chiropractic College outpatient teaching clinics in lowa and Illinois (May 2017). Descriptive statistics were used to analyze respondent characteristics and prevalence of SBT-calculated risk subgroups. Binary logistic regression analysis was used to examine the relationship between respondent characteristics and SBT scores (including psychological subscores).

Results: Of 550 respondents, 496 completed the SBT; 392 (79\%) scored low-risk, 81 (16\%) medium-risk, and $23(5 \%)$ high-risk. Mean (SD) age was 44.8 (15.9), 56.9\% were female, 88.2\% white, 62.6\% employed, mean current pain was 2.9 (2.1) out of 10, and $62 \%$ reported symptom duration $>3$ months. Eighteen percent of respondents reported anxious thoughts, 32\% low mood, $41 \% \geq 1$ and 21\% $\geq 3$ SBT psychological risk factors. Respondents reporting higher average pain $(\mathrm{OR}=1.8[1.4,2.3])$ and pain severity $(\mathrm{OR}=1.3[1.0$ to 1.6]) were more likely to score with medium or high risk. Respondents reporting mid back versus low back pain $(\mathrm{OR}=0$. $2[0.1,0.7])$, and those employed less than full-time versus full-time $(0.2[01,0.5])$ were less likely to score with medium or high risk. Respondents reporting higher average pain were more likely to report $\geq 1$ psychological factor $(\mathrm{OR}=1.8[1.5,2.0])$. Respondents employed part-time were less likely to report $\geq 1$ psychological factor than those employed full-time ( $\mathrm{OR}=0.4[0.2,0.7])$.

Conclusion: The sample surveyed was less likely to score with medium or high risk for back pain disability than previous samples studied, perhaps due to differences in study design and sample characteristics. Rates of low mood and anxious thoughts indicate a need for future research to explore psychological factors among persons seeking chiropractic care.
\end{abstract}

Keywords: STarT Back screening tool, Back pain, Chiropractic, Disability, Back pain disability, Chronic pain, Low Back pain, Biopsychosocial

\footnotetext{
* Correspondence: yasmeen-khan@uiowa.edu

${ }^{1}$ Parker University, 2500 Walnut Hill Lane, Dallas, TX 75229, USA

Full list of author information is available at the end of the article
}

(c) The Author(s). 2019 Open Access This article is distributed under the terms of the Creative Commons Attribution 4.0 International License (http://creativecommons.org/licenses/by/4.0/), which permits unrestricted use, distribution, and reproduction in any medium, provided you give appropriate credit to the original author(s) and the source, provide a link to the Creative Commons license, and indicate if changes were made. The Creative Commons Public Domain Dedication waiver (http://creativecommons.org/publicdomain/zero/1.0/) applies to the data made available in this article, unless otherwise stated. 


\section{Background}

Spinal pain is one of the most costly and prevalent conditions worldwide [1-4]. Because of the interplay between musculoskeletal and psychological conditions, spinal pain-back pain in particular-can be considered a biopsychosocial phenomenon [5-10]. While some evidence suggests that psychological factors do not predict outcomes in chiropractic settings [11], anxiety, depression, fear-avoidance behavior, low expectations of recovery, and catastrophizing have been shown to predict poor outcomes in medical and chiropractic settings [6, 7, 12-21]. Furthermore, psychological factors are associated with the transition from acute to chronic back pain $[6,12,22]$. Identifying both biomedical and psychological factors associated with spinal pain facilitates a more comprehensive understanding of patients' clinical needs [23, 24].

The STarT Back Screening Tool (SBT) is a 9-item questionnaire that screens for physical and psychological risk factors for back pain disability $[25,26]$. The SBT was developed within a cohort of patients seeking primary medical care for low back pain (LBP) of any duration (acute, subacute and chronic), with or without referred pain to the lower extremity [25]. Data obtained from the SBT have been studied in several patient populations and exhibit reliability in detecting biopsychosocial risk factors for back pain disability (pain severity, physical functioning, and psychological factors) [20, 27, 28]. More specifically, SBT items inquire about presence of neck or shoulder pain, pain referral to the leg, pain bothersomeness, physical functioning (dressing and walking), fear-avoidance beliefs, catastrophizing, low mood (loss of interest or pleasure), and anxious thoughts $[29,30]$. Scores-ranging from 0 to 9 -categorize patients with low, medium or high risk for developing back pain disability [29]. The purpose of using the SBT in clinical settings is to screen for risk of developing persistent disabling pain among persons experiencing an episode of LBP. The SBT also identifies patient risk subgroups for targeted treatments (Subgroups for Targeted Treatment, or STarT), meaning that specific treatment plans are matched to SBT score category [29].

Studies report the prevalence of SBT risk subgroups among patients seeking chiropractic care outside of the US [31-35], but similar analyses in US chiropractic patient populations have not been reported. In previous studies, the SBT was shown to be feasibly incorporated into Danish private practice chiropractic clinics [32] and exhibited construct validity in the European populations studied $[31,36]$. One study indicated that SBT scores are less predictive among persons with an episode duration under 2 weeks, a characteristic more common among patients seeking chiropractic versus medical care [35]. Another study demonstrated that SBT scores were labile within the first few days after an initial chiropractic visit, also affecting its predictive utility [34]. The wide variety of respondent characteristics and SBT risk profiles among cohorts studied suggests a need for further inquiry.

Nearly 34 million people (14\% of the population) in the US sought chiropractic care in 2015 alone [37]. Yet SBT score category prevalence and SBT-informed psychological risk profiles among US patients seeking chiropractic care is still unclear. Therefore, the primary purpose of this study is to report the prevalence of low, medium, and high-risk for back pain disability in adult patients receiving chiropractic care in the US, measured by the SBT. The secondary purpose is to examine the relationship between respondent characteristics and SBT scores (including responses to SBT psychological distress items).

\section{Methods \\ Study design and setting}

This study used a cross-sectional survey design. The Palmer College of Chiropractic Institutional Review Board approved this study and granted "exempt" status in April, 2017. Respondents did not share identifying information on the paper surveys. The paper survey was administered at 4 Palmer College of Chiropractic outpatient clinics in 3 locations in Moline, IL and Davenport, IA, US, from May 1-31, 2017. Clinic staff provided eligible participants with a paper copy of the survey as they presented for scheduled office visits. Surveys contained written directions for completion and were comprised of the 9-item SBT and 15 demographic questions to identify sample characteristics. Respondents completed surveys independently and returned them to the front desk staff upon completion.

\section{Participants and recruitment}

Eligible participants were 18 years or older, receiving care at one of the outpatient clinics, and were willing to complete the survey. Participants were instructed to complete the survey only once. To maximize response rate and mitigate coverage error, a study team member (YK) trained office staff to administer surveys as patients presented to the clinics and to make every attempt to supply all eligible patients with a survey. The study team member visited each clinic 1-3 times per week to confirm adherence to study protocols, collect completed surveys, and address questions or concerns.

In line with study aims, any adult presenting to participating clinics during the data collection time frame was permitted to complete the survey, regardless of previous duration of chiropractic care or primary symptom. Although the SBT was designed to be implemented during an acute LBP episode [30], SBT items identify physical 
and psychological factors that are also clinically pertinent in the management of chronic LBP [38]. In addition, the SBT includes items relevant to both neck and back pain (pain in the shoulder or neck and referred pain to the leg). By including participants of any care duration or symptom region, data collected in the present study reflect the clinical characteristics of a broad range of patients receiving care at US chiropractic teaching clinics.

\section{Statistical analysis}

The primary outcome of interest was the prevalence of each of the 3 SBT risk subgroups. Secondary outcomes included the prevalence of positive responses to each SBT item, the odds of achieving a high-risk score, the odds of achieving a medium or high-risk score (MHRS), and the odds of reporting $\geq 1$ SBT psychological risk factor. The initial study protocol included an analysis of the odds of scoring with high-risk rather than a collapsed medium- and high-risk subgroup. However, the study sample did not contain a large enough pool of respondents with high-risk scores to produce a statistically valid analysis [39].

By collapsing the medium- and high-risk subgroups, the sample size requirement was met, while maintaining clinically relevant results. For example, according to the SBT matched treatment protocol, persons who score with low risk do not receive any further care, while those scoring with medium or high risk are referred for additional treatment. Understanding which patient characteristics may be related to the need for further care is clinically pertinent. The logistic regression analysis of the medium- and high-risk subgroup outcome variable was expected to provide clinical insight and expand the research base related to caring for patients at risk for future disabling pain.

The rationale for exploring factors related to reporting at least 1 psychological factor was similar. For example, previous studies suggest that individual psychological factors alone may contribute to risk for chronic back pain $[6,14]$. Because the presence of individual psychological risk factors may affect treatment decisions, the prevalence and likelihood of reporting at least 1 factor is clinically relevant. In line with the study aims, both logistic regression analyses were expected to provide a better understanding of the characteristics of patients receiving chiropractic care in the US and provide a foundation for future research.

We used a double-key entry process to verify data. Analysis of datasets utilized Statistical Package for the Social Sciences (SPSS) version 23.0 (IBM, Chicago, IL). Descriptive statistics using counts and percentages were generated to report demographic characteristics of the total sample, across SBT risk subgroups, and for each of the 9 SBT items.
We performed 3 binary logistic regression analyses. Respondent SBT scores were dichotomized into those who: 1) scored with high risk versus those who did not; 2) those who scored with medium-to-high risk versus those who did not; and 3) those who reported $\geq 1$ psychological risk factor versus those who did not. Exploratory data analysis tested for bivariate interactions between continuous and categorical explanatory variables (Table 1 lists the 13 explanatory variables used in these analyses). Logistic regression model assumptions (a dichotomized outcome variable and independent error terms) were validated. Observations with missing values were not included in this data analysis.

We used a simple binary logistic regression analysis to compute odds ratios (ORs) and 95\% confidence intervals for the likelihood of achieving a high-risk score, a medium-to-high-risk score, and reporting $\geq 1$ psychological risk factor, based on each explanatory variable.

We considered individual explanatory variables and interactions that produced statistically significant ORs $(p \leq 0.1)$ for inclusion in multiple logistic regression analyses. All variables that had a statistically significant $(p \leq 0.05)$ contribution to the odds for each outcome variable were considered for inclusion in the final models.

\section{Results}

Of the 599 eligible patients presenting in May, 2017, 550 completed surveys, resulting in a $91.8 \%$ response rate. Four hundred ninety-six respondents completed all 9 SBT items (response rate $=90 \%$ of respondents) . Table 1 summarizes the study population's baseline demographic and clinical characteristics. The mean (SD) age of the sample was 44.8 (15.9) and the mean current pain reported on a 0-10 scale was 2.9 (2.1). The majority of the sample was female $(57 \%)$, Caucasian (88\%), and employed part- or full-time (63\%). Twenty-five percent of respondents selected a primary symptom of LBP, $15 \%$ neck pain, and $10 \%$ mid back pain, 27\% more than 1 pain region, and 24\% "other" indicating a reason for presenting to the clinic that was not listed. The majority (62\%) of respondents reported an episode duration over 12 weeks. Most (62\%) also reported having received chiropractic care for over 12 weeks for their presenting condition.

\section{STarT Back subgroup and item prevalence}

The primary aim of this study was to report the prevalence of each SBT risk subgroup. Of the 496 respondents who completed all SBT items, 392 (79.0\%) achieved a low-risk SBT score, 81 (16.3\%) a medium-risk score, and $23(4.6 \%)$ a high-risk score. Among persons reporting a primary symptom of LBP $(n=127), 89(70 \%)$ scored with low risk, 30 (24\%) scored with medium risk, 
Table 1 Descriptive analysis of demographic variables for whole study sample and across STarT Back Tool risk subgroups for patients presenting for chiropractic care $(n=550)$

\begin{tabular}{|c|c|c|c|c|c|c|}
\hline \multirow[t]{2}{*}{ Variables } & \multirow{2}{*}{$\begin{array}{l}\mathrm{n} \text { for } \\
\text { whole } \\
\text { sample }\end{array}$} & \multirow{2}{*}{$\begin{array}{l}\text { Whole } \\
\text { Sample }\end{array}$} & \multirow[b]{2}{*}{$\begin{array}{l}\mathrm{n} \text { for } \\
\text { select } \\
\text { variable } \\
\text { across } \\
\text { STarT } \\
\text { Back } \\
\text { subgroup }\end{array}$} & \multicolumn{3}{|c|}{ STarT Back Risk Subgroup } \\
\hline & & & & Low & Medium & High \\
\hline Age-mean (SD) & 529 & $44.8(15.9)$ & & $42.4(15.4)$ & $50.9(17.1)$ & $45.1(13.6)$ \\
\hline Sex-n (\%) & 536 & & 486 & & & \\
\hline Male & & $230(42.9)$ & & $166(43.2)$ & $33(41.3)$ & $11(50.0)$ \\
\hline Female & & $305(56.9)$ & & $217(56.5)$ & $47(58.8)$ & $11(50$ \\
\hline Prefer not to answer & & $0(0.0)$ & & $0(0.0$ & $0(0.0)$ & $0(0.0)$ \\
\hline Prefer to self-describe & & $1(0.2)$ & & $1(0.3)$ & $0(0.0)$ & $0(0.0)$ \\
\hline Race-n (\%) & 524 & & 475 & & & \\
\hline Native American or Alaskan Native & & $2(0.4)$ & & $1(0.3)$ & $1(0.3)$ & $0(0.0)$ \\
\hline Asian & & $6(1.1)$ & & $3(0.8)$ & $2(2.5)$ & $0(0.0)$ \\
\hline Black or African American & & $27(5.2)$ & & $13(3.5)$ & $6(7.5)$ & $4(18.2)$ \\
\hline Native Hawaiian or Pacific Islander & & $1(0.2)$ & & $1(0.3)$ & $0(0.0)$ & $0(0.0)$ \\
\hline White & & $462(88.2)$ & & $337(90.3)$ & $67(83.8)$ & $16(72.7)$ \\
\hline$>1$ race & & $26(5.0)$ & & $18(4.8)$ & $4(5.0)$ & $2(9.1)$ \\
\hline Ethnicity-n (\%) & 498 & & 453 & & & \\
\hline Hispanic/Latino & & $33(6.6)$ & & $23(6.4)$ & $6(8.0)$ & $2(10.5)$ \\
\hline Non-Hispanic/non-Latino & & $465(93.4)$ & & $336(93.6)$ & $69(92.0)$ & 17 (89.5) \\
\hline Employment status-n (\%) & 511 & & 468 & & & \\
\hline Full-time & & $251(49.1)$ & & $201(54.0)$ & $23(31.1)$ & $11(50.0)$ \\
\hline Part-time & & $69(13.5)$ & & $54(14.5)$ & $9(12.2)$ & $1(4.5)$ \\
\hline Full-time student & & $64(11.6)$ & & $53(14.2)$ & $5(6.8)$ & $1(4.5)$ \\
\hline Unemployed & & $127(24.9)$ & & $64(17.2)$ & $37(50)$ & $9(40.9)$ \\
\hline Occupation-n (\%) & 514 & & 467 & & & \\
\hline Educator & & $40(7.8)$ & & $36(9.7)$ & $2(2.7)$ & $0(0.0)$ \\
\hline Health care professional & & $61(11.9)$ & & $48(12.9)$ & $6(8.1)$ & $1(0.0)$ \\
\hline Active duty military & & $36(7.0)$ & & $29(7.8)$ & $4(5.4)$ & $0(0.0)$ \\
\hline Law enforcement & & $2(0.4)$ & & $0(0.0)$ & $1(1.4)$ & $1(4.5)$ \\
\hline Computer technology & & $16(3.1)$ & & $12(3.2)$ & $1(1.4)$ & $0(0.0)$ \\
\hline Transportation industry & & $9(1.8)$ & & $8(2.2)$ & $1(1.4)$ & $0(0.0)$ \\
\hline Agriculture & & $10(1.9)$ & & $5(1.3)$ & $2(2.7)$ & $0(0.0)$ \\
\hline Other & & $340(66.1)$ & & $233(62.8)$ & $57(77.0)$ & $20(90.9)$ \\
\hline Marital status-n (\%) & 534 & & 484 & & & \\
\hline Never been married & & $147(27.5)$ & & $106(27.7)$ & $19(24.1)$ & $6(27.3)$ \\
\hline Married/living with significant other & & $294(55.1)$ & & $221(57.7)$ & $45(57.0)$ & $6(27.3)$ \\
\hline Divorced & & $75(14.0)$ & & $48(12.5)$ & $11(13.9)$ & $8(36.4)$ \\
\hline Widow & & $18(3.4)$ & & $8(2.1)$ & $4(5.1)$ & $2(9.1)$ \\
\hline Primary symptom area-n (\%) & 549 & & 495 & & & \\
\hline Low back pain & & $136(24.8)$ & & $89(22.8)$ & $30(37.0)$ & $8(34.8)$ \\
\hline Neck pain & & $80(14.6)$ & & $66(16.9)$ & $4(4.9)$ & $2(8.7)$ \\
\hline Mid-back pain & & $52(9.5)$ & & $42(10.7)$ & $4(4.9)$ & $2(8.7)$ \\
\hline
\end{tabular}


Table 1 Descriptive analysis of demographic variables for whole study sample and across STarT Back Tool risk subgroups for patients presenting for chiropractic care $(n=550)$ (Continued)

\begin{tabular}{|c|c|c|c|c|c|c|}
\hline \multirow[t]{2}{*}{ Variables } & \multirow{2}{*}{$\begin{array}{l}\mathrm{n} \text { for } \\
\text { whole } \\
\text { sample }\end{array}$} & \multirow{2}{*}{$\begin{array}{l}\text { Whole } \\
\text { Sample }\end{array}$} & \multirow[b]{2}{*}{$\begin{array}{l}\mathrm{n} \text { for } \\
\text { select } \\
\text { variable } \\
\text { across } \\
\text { STarT } \\
\text { Back } \\
\text { subgroup }\end{array}$} & \multicolumn{3}{|c|}{ STarT Back Risk Subgroup } \\
\hline & & & & Low & Medium & High \\
\hline Other & & $132(24.0)$ & & $109(27.9)$ & $10(12.3)$ & $0(0.0)$ \\
\hline$>1$ region & & $149(27.1)$ & & $85(21.7)$ & $33(40.7)$ & $11(47.8)$ \\
\hline Current episode duration-n (\%) & 548 & & 494 & & & \\
\hline $\mathrm{n} / \mathrm{a}$ & & $46(8.4)$ & & $38(9.7)$ & $2(2.5)$ & $0(0.0)$ \\
\hline$<2$ weeks & & $60(10.9)$ & & $46(11.8)$ & $7(8.8)$ & $4(17.4)$ \\
\hline 2-4 weeks & & $43(7.8)$ & & $32(8.2)$ & $3(3.8)$ & $2(8.7)$ \\
\hline $5-12$ weeks & & $59(10.8)$ & & $40(10.2)$ & $10(12.5)$ & $2(8.7)$ \\
\hline 13 weeks - 1 year & & $71(13.0)$ & & $52(13.3)$ & $12(15.0)$ & $2(8.7)$ \\
\hline$>1$ year & & $269(49.1)$ & & $183(46.8)$ & $46(57.5)$ & $13(56.5)$ \\
\hline Chiropractic care duration-n (\%) & 547 & & 493 & & & \\
\hline n/a or have not received chiropractic care for condition & & $52(9.4)$ & & $39(7.2)$ & $4(4.9)$ & $1(4.3)$ \\
\hline$<2$ weeks & & $41(7.5)$ & & $29(7.5)$ & $5(6.2)$ & $2(8.7)$ \\
\hline 2-4 weeks & & $49(9.0)$ & & $34(8.7)$ & $9(11.1)$ & $1(4.3)$ \\
\hline 5 to 12 weeks & & $67(12.2)$ & & $50(12.9)$ & $10(12.3)$ & $2(8.7)$ \\
\hline 13 weeks - 1 year & & $78(14.3)$ & & $58(14.9)$ & $9(11.1)$ & $4(17.4)$ \\
\hline$>1$ year & & $260(47.3)$ & & $179(46.0)$ & $44(54.3)$ & $13(56.5)$ \\
\hline Average pain in past 2 weeks-mean (SD) & 547 & $3.6(2.2)$ & & $3.0(1.8)$ & $5.5(1.8)$ & $7.3(1.5)$ \\
\hline Most severe pain in past 2 weeks-mean (SD) & 547 & $5.1(2.6)$ & & $4.4(2.4)$ & $7.2(1.8)$ & $8.7(1.3)$ \\
\hline Current pain-mean (SD) & 530 & $2.9(2.1)$ & & $2.3(1.8)$ & $4.6(1.8)$ & $5.9(2.6)$ \\
\hline
\end{tabular}

and $8(6 \%)$ scored with high risk. Among persons with an episode duration under 2 weeks $(n=57), 46(81 \%)$ scored with low risk, 7 (12\%) with medium risk, and 4 (7\%) with high risk.

Secondary aims were to report the mean SBT score and the proportion of positive responses to each SBT item. Table 2 summarizes the descriptive statistics for SBT total and item scores. The mean (SD)
SBT total score was 2.1 (1.9). Of respondents completing the SBT, 32\% reported low mood (loss of interest), $18 \%$ reported anxious thoughts, $17 \%$ reported 'very much' or 'extremely' bothersome pain, $11 \%$ reported catastrophic thoughts, and $9 \%$ reported fear-avoidance beliefs.

The Odds of Scoring in a Medium-to-High-Risk Group and Reporting Psychological Risk Factors.

Table 2 Mean STarT Back scores and descriptive characteristics by domain

\begin{tabular}{|c|c|c|}
\hline STarT Back total score-mean (SD) & $n=496$ & $2.1(1.9)$ \\
\hline STarT Back-5 psychological distress subscore-mean (SD) & $n=506$ & $0.8(1.2)$ \\
\hline Referred pain to leg $(Y)-n(\%)$ & $n=539$ & $94(17.4)$ \\
\hline Shoulder or neck pain (Y)-n (\%) & $n=544$ & $412(75.7)$ \\
\hline Physical Function: Only walked short distances (Y)-n (\%) & $n=537$ & $99(18.4)$ \\
\hline Physical Function: Dressed more slowly (Y)-n (\%) & $n=537$ & $100(18.6)$ \\
\hline Fear-avoidance: Not safe to be active (Y)-n (\%) & $n=532$ & $48(8.7)$ \\
\hline Anxiety: Worrying thoughts (Y)-n (\%) & $n=536$ & $95(17.7)$ \\
\hline Catastrophizing: Back pain is terrible and never going to get better $(\mathrm{Y})-\mathrm{n}(\%)$ & $n=537$ & $57(10.6)$ \\
\hline Depression: Not enjoying things I used to (Y)-n (\%) & $n=531$ & $168(31.6)$ \\
\hline Bothersomeness (very much to extremely)-n (\%) & $n=538$ & $91(16.5)$ \\
\hline
\end{tabular}


Another secondary aim was to report which demographic and clinical characteristics contributed to the odds of scoring in the high-risk group, the medium-tohigh-risk group, and reporting $\geq 1$ psychological risk factor. However, we did not obtain a large enough pool of high-risk respondents to conduct a sound regression analysis, hence the post hoc change to a pooled medium-to-high risk group. Tables 3 and 4 report ORs and $95 \%$ confidence intervals for the odds of achieving a MHRS and reporting $\geq 1$ psychological risk factor.

One hundred and four (20.9\%) respondents were categorized with MHRSs. Average pain rating in the past 2 weeks (on a 0 to 10 scale), most severe pain rating the past 2 weeks (on a 0 to 10 scale), primary symptom, and employment status correctly predicted a MHRS $89 \%$ of the time. Respondents reporting higher average pain intensity were more likely to achieve a MHRS (OR $=1.8$ $[1.4,2.3, p<0.001$; Table 3). Respondents reporting higher rates of the most severe pain intensity in the past 2 weeks were more likely to achieve a MHRS (OR $=1.3$ $[1.0,1.6], p=0.02)$.

Respondents reporting neck pain as a primary symptom reported approximately the same likelihood of achieving a MHRS compared to those reporting LBP. Those reporting LBP were more likely to achieve a MHRS than those reporting mid back pain, more than 1 primary symptom category, and those who answered "other." However, mid back pain was the only symptom category with a statistically significant effect on the odds of a MHRS, relative to the LBP referent category $(\mathrm{OR}=0.2[0.1,0.7], p=0.02)$. In

Table 3 Odds ratios for scoring in the medium or high-risk STarT Back subgroups based on select explanatory variables ${ }^{a}$

\begin{tabular}{llll}
\hline Explanatory Variable & OR & $\begin{array}{l}\text { 95\% Confidence } \\
\text { Interval }\end{array}$ & $p$-value \\
\hline $\begin{array}{l}\text { Average pain in past 2 } \\
\text { weeks }\end{array}$ & $1.8^{c}$ & $(1.4,2.3)$ & $<0.001$ \\
$\begin{array}{l}\text { Most severe pain in past 2 } \\
\text { weeks }\end{array}$ & $1.3^{c}$ & $(1.0,1.6)$ & 0.02 \\
$\begin{array}{l}\text { Primary symptom area } \\
\text { Low back pain }\end{array}$ & - & - & \\
Neck pain & 1.0 & $(0.5,2.1)$ & 1.00 \\
Mid-back & $0.2^{c}$ & $(0.1,0.7)$ & 0.02 \\
$>1$ area of complaint & 0.4 & $(0.1,1.1)$ & 0.11 \\
Other & 0.3 & $(0.1,1.3)$ & 0.07 \\
Employment status & & & \\
Full-time & - & - & 0.001 \\
Part-time & $0.2^{c}$ & $(0.1,0.5)$ & 0.01 \\
Full-time student & $0.3^{c}$ & $(0.1,0.7)$ & 0.02 \\
Unemployed & $0.0^{c}$ & $(0.1,0.7)$ & \\
\hline
\end{tabular}

${ }^{\mathrm{a}}$ Hosmer and Lemeshow Test $p$-value $=0.799$

${ }^{\mathrm{b}}$ Referent category

indicates statistical significance at alpha $=0.05$ level
Table 4 Odds of reporting $\geq 1$ psychological risk factor based on select explanatory variables ${ }^{a}$

\begin{tabular}{llll}
\hline Explanatory Variable & OR & $95 \%$ Confidence Interval & $p$-value \\
\hline $\begin{array}{l}\text { Average pain in past } \\
2 \text { weeks }\end{array}$ & $1.8^{c}$ & $(1.5,2.0)$ & $<0.001$ \\
Employment status & & & \\
Full-time & - & - & 0.001 \\
Part-time & $0.4^{c}$ & $(0.2,0.7)$ & 0.05 \\
Full-time student & 0.5 & $(0.2,1.0)$ & 0.62 \\
Unemployed & 0.8 & $(0.4,1.9)$ &
\end{tabular}

${ }^{\mathrm{a}}$ Hosmer and Lemeshow Test $\mathrm{p}$-value $=0.950$

${ }^{\mathrm{b}}$ Referent category

indicates statistical significance at alpha $=0.05$ level

other words, reporting a primary symptom of low back pain instead of mid back pain was associated with an increased odds of achieving a MHRS.

Respondents who were employed part-time, were full-time students, or were unemployed were less likely to achieve a MHRS compared to those who were employed full-time $(\mathrm{OR}=0.2[0.1,0.5], p<0.001 ; \mathrm{OR}=0.3[0.1,0.7]$, $p=0.01 ; \mathrm{OR}=0.0[0.1,0.7], p=0.02)$. In other words, full-time working status was associated with increased odds of achieving a MHRS.

Forty-one percent of respondents reported $\geq 1$ SBT-identified psychological risk factor, with low mood reported most commonly. Average pain intensity in the past 2 weeks and employment status correctly predicted the presence of $\geq 1$ psychological condition $74 \%$ of the time. Respondents with higher average pain intensity were significantly more likely to report $\geq 1$ psychological risk factor $(\mathrm{OR}=1.8[1.5,2.0], p<0.001)$; Table 4). Respondents who were part-time employed, full-time students, or unemployed were less likely to report $\geq 1$ psychological risk factor compared to those were employed full-time. However, part-time employment status was the only employment category that had a statistically significant effect on the odds of reporting $\geq 1$ psychological risk factor, relative to the full-time status referent category $(\mathrm{OR}=0.4[0.2$, 0.7], $p=0.001$ ).

\section{Discussion}

To our knowledge, this is the first study to report the prevalence of SBT-designated risk subgroups among persons in the US seeking chiropractic care. Compared with chiropractic patient cohorts in previous studies, respondents in this study achieved MHRSs at lower rates [3134]. Most previously published studies employing the SBT in chiropractic settings were conducted within patient cohorts reporting an acute episode of non-specific LBP [31, 32, 34] whereas the present study allowed inclusion of all adult patients regardless of episode duration or primary symptom. Overall, $91 \%$ of respondents had received some form of chiropractic care for their 
condition at the time of data collection, and less than half reported a primary symptom of LBP. Nevertheless, among respondents with an episode duration under 2 weeks, a chiropractic care duration under 2 weeks, and LBP, the prevalence of MHRSs was lower than rates reported in previous chiropractic cohorts [31-35]. This indicates that these factors alone cannot explain the lower rates of MHRSs in the present study.

In the present study, care duration was not significantly associated with a MHRS. On the other hand, reporting low back relative to mid back pain was associated with an increased likelihood of a MHRS. Furthermore, among respondents reporting a primary symptom of LBP (versus neck or mid back pain), the SBT score distribution more closely matched distributions reported in previous chiropractic settings (Present study, primary symptom LBP: low $=70 \%$, medium $=24 \%$, high $=6 \%$; Kongsted et al., 2011: low $=59 \%$, medium $=29 \%$, high $=$ $11 \%$; Morso et al., 2016: low $=52 \%$, medium $=39 \%$, high $=10 \%)[32,35,40]$. Similarly, among respondents reporting more than 1 region of pain (versus neck or mid back pain), SBT score distributions more closely matched those reported in previous studies (low $=66 \%$, medium $=26 \%$, high $=9 \%$; Table 1 ). However, among respondents reporting LBP or more than 1 symptom, and within the overall sample, the prevalence of MHRSs was lower than in previous chiropractic patient cohorts [40]. Based on the study design and data collected, it is unclear how differences between the current study sample and those described in previous studies may have contributed to the lower prevalence of MHRSs in this sample.

Despite a lower prevalence of MHRSs, responses indicating low mood, anxious thoughts, catastrophizing, and pain bothersomeness were common (Table 2). For example, $32 \%$ of respondents reported low mood (loss of interest or pleasure), $19 \%$ reported anxious thoughts, $16 \%$ reported catastrophic thoughts, and $22 \%$ reported a perception that their pain was 'very much' or 'extremely' bothersome. Although individual SBT items are not diagnostic of mental health conditions, $19 \%$ of respondents reported having anxious thoughts, consistent with prevalence rates for anxiety disorders among US adults (19\%) [41]. On the other hand, $32 \%$ of respondents reported low mood (loss of interest or pleasure) compared to $6.7 \%$ of the US adult population reporting 1 or more depressive episodes in a given year [42]. A loss of pleasure may be more common than clinical depression itself, however data collected in this study cannot explain the high rates of low mood among respondents.

Results of this study indicate that respondent pain severity was associated with reporting 1 or more SBT psychological risk factors. Average pain in the past 2 weeks was significantly associated with increased odds of reporting $\geq 1$ SBT psychological risk factor, and achieving a MHRS. This result confirms previous findings suggesting that higher pain scores are associated with higher SBT total and SBT-5 psychological distress subscores [35, 43, 44].

The relationship between full-time employment status and SBT risk category is also notable and worth future scientific investigation. There may be a relationship between specific vocational activities and SBT score over time. Because a substantial proportion of respondents had received prior chiropractic care, results of the logistic regression analysis may not indicate whether employment status, pain severity, and pain location are related to an initial high risk for persistent back pain disability. Instead, these analyses may indicate which demographic factors are associated with a change in SBT scores over time, or the maintenance of relatively high scores over time. It may be noted that the majority of respondents scoring with high risk $(57 \%)$ reported receiving care for more than 1 year (Table 1). The analyses conducted for the present study cannot explain the association between full-time employment status, pain severity or location and SBT item responses.

This study has several limitations inherent to its design. Due to the observational study design, causative factors influencing SBT scores cannot be identified, and study results must be interpreted conservatively. Also, there may have been differences in SBT scores and demographic characteristics between respondents and non-respondents, leading to non-response error. The high response rate $(92 \%$ overall, $83 \%$ completing the entire SBT) mitigates this potential to a large extent. Next, despite attempts to minimize measurement error, patient self-reporting can limit data accuracy and validity. Coverage error was possible because the survey was administered at clinics in 1 geographic location. Therefore, study results may not be generalizable to all US patients seeking chiropractic care.

The SBT is a screening instrument designed to be employed at the onset of care for a LBP episode. Yet, most respondents in the present study had already received some form of chiropractic care for their condition at the time of data collection. Because previous literature demonstrates that SBT scores are labile both in the initial stages and over the course of chiropractic care [31, 34], the greater prevalence of persons having received chiropractic care may have contributed to the lower rates of MHRSs relative to previous studies in chiropractic settings. Similarly, less than half of respondents reported a primary symptom of LBP. As a result, the SBT subgroup classifications may not be predictive of outcomes in some respondents. Analyses conducted for the present study cannot explain the lower prevalence of MHRSs relative to previous studies conducted in 
chiropractic settings [31-35], although respondents reporting LBP were more likely to score with medium or high risk. Because there are few studies of this kind, similar research efforts focused on US private practice settings could provide additional insight into the characteristics of a broad range of chiropractic patients.

\section{Clinical implications}

Results suggest that a clinician in the study setting might expect 1 out of every 2 to 3 patients to be at risk for depression, 1 out 4 to 5 to be at risk for anxiety, and approximately 1 out of 6 patients to be at risk for potentially catastrophizing thought patterns that may impede patient progress. Doctors of chiropractic, like many other primary contact healthcare providers, may consider augmenting their knowledge about, and preparedness for, managing care for patients experiencing fear-avoidance beliefs, catastrophizing, depression, anxiety, and perceived pain bothersomeness [45-47].

Previous trials examining the use of STarT Back matched treatment plans suggest that healthcare providers who work with back pain-related conditions can improve patient outcomes by implementing cognitive behavioral approaches to assist in overcoming psychological barriers to recovery $[29,48]$. Some chiropractic clinicians currently implement strategies to help patients overcome maladaptive cognitive and emotional responses to pain, such as graded exposure and behavioral interventions [49]. The prevalence of psychological risk factors among respondents in this study suggests the importance of clinician awareness of the common need for such interventions.

\section{Conclusion}

Seventy-nine percent of respondents scored with low risk for developing back pain disability, $21 \%$ scored medium-to-high risk for developing back pain disability, and $41 \%$ reported at least 1 SBT psychological risk factor. The SBT is a screening tool and cannot be used to diagnose mental health conditions. However, a relatively high prevalence of respondents indicating low mood and anxious thoughts supports the need for future research efforts aimed at quantifying the prevalence of common psychological risk factors and mental health conditions among US patients seeking chiropractic care. Survey results suggest that clinicians at US chiropractic teaching clinics should develop, or continue implementing, management pathways for patients experiencing specific types of psychological distress. Future studies examining how SBT-informed tailored treatment plans influence pain, disability, and quality of life outcomes are also needed.

\section{Abbreviations}

LBP: Low back pain; MHRS: Medium-to-high-risk score; SBT: STarT Back Screening Tool

\section{Acknowledgements}

We would like to thank Lance Corber, MSITM, for his assistance in data entry and data management.

\section{Funding}

This project was supported by a graduate research assistantship funded in part by the Palmer Center for Chiropractic Research.

Availability of data and materials

The datasets used and/or analyzed during the current study are available from the corresponding author on reasonable request.

\section{Authors' contributions}

YK was responsible for the conception, development and execution of this project, the data collection and analysis, and drafting this manuscript. DL was responsible for the development of the project and drafting this manuscript. RV was responsible for development and execution of this project and drafting this manuscript. DD was responsible for assisting with data analysis and drafting of the manuscript. All authors read and approved the final manuscript.

\section{Authors' information}

YK. This project was conducted as part of a Master of Science in Clinical Research degree program at Palmer College of Chiropractic. She is currently an independent chiropractic clinician and full-time Master of Art student at the

University of lowa, studying Rehabilitation and Mental Health Counseling.

DL was the Graduate Advisory Committee Chair for project. He is the Senior

Director for the Center for Teaching and Learning and Continuing Education, Parker University, Dallas TX.

RV was a member of the Graduate Advisory Committee for this project. He is an Associate Professor and Senior Clinician, Palmer Center for Chiropractic Research, Davenport IA

DD was a member of the Graduate Advisory Committee for project. He is the Senior Director for Institutional Research and Effectiveness, and the Human Research Protections Administrator for the Institutional Review Board at Palmer College of Chiropractic, Davenport IA.

Ethics approval and consent to participate

Ethics approval was obtained from the Palmer College of Chiropractic Institutional Review Board. This study was granted "exempt" status.

Consent for publication

Not applicable.

Competing interests

The authors declare that they have no competing interests.

\section{Publisher's Note}

Springer Nature remains neutral with regard to jurisdictional claims in published maps and institutional affiliations.

\section{Author details}

${ }^{1}$ Parker University, 2500 Walnut Hill Lane, Dallas, TX 75229, USA. Palmer Center for Chiropractic Research, 741 Brady Street, Davenport, IA 52803, USA.

${ }^{3}$ Palmer College of Chiropractic, 1000 Brady Street, Davenport, IA 52803, USA.

Received: 23 January 2018 Accepted: 14 December 2018

Published online: 15 January 2019

\section{References}

1. Global Burden of Disease Study 2013 Collaborators. Global, regional, and national incidence, prevalence, and years lived with disability for 301 acute and chronic diseases and injuries in 188 countries, 19902013: a systematic analysis for the Global Burden of Disease Study 2013. Lancet. 2015:386:743-800.

2. March L, Smith EUR, Hoy DG, Cross MJ, Sanchez-Riera L, Blyth F, et al. Burden of disability due to musculoskeletal (MSK) disorders. Best Pract Res Clin Rheumatol. 2014;28:353-66. 
3. Dagenais S, Caro J, Haldeman S. A systematic review of low back pain cost of illness studies in the United States and internationally. Spine J. 2008;8:8-20.

4. Gore M, Sadosky A, Stacey BR, Tai K-S, Leslie D. The burden of chronic low back pain: clinical comorbidities, treatment patterns, and health care costs in usual care settings. Spine. 2012;37:E668-77.

5. Waddell G. Biopsychosocial analysis of low back pain. Baillieres Clin Rheumatol. 1992;6:523-57.

6. Murphy DR, Hurwitz EL. The usefulness of clinical measures of psychologic factors in patients with spinal pain. J Manip Physiol Ther. 2011;34:609-13.

7. Burton AK, Erg E. Back injury and work loss. Biomechanical and psychosocial influences. Spine. 1997;22:2575-80.

8. Peters ML, Vlaeyen JWS, Weber WEJ. The joint contribution of physical pathology, pain-related fear and catastrophizing to chronic back pain disability. Pain. 2005;113:45-50.

9. Sterling M. Balancing the "bio" with the psychosocial in whiplash associated disorders. Man Ther. 2006;11:180-1.

10. Waddell G. The back pain revolution. 1st ed. New York: Churchill Livingstone; 1999. 438 p.

11. Ailliet $L$, Rubinstein SM, Hoekstra T, van Tulder MW, de Vet HCW. Adding psychosocial factors does not improve predictive models for people with spinal pain enough to warrant extensive screening for them at baseline. Phys Ther. 2016;96:1179-89.

12. Del Campo MT, Romo PE, de la Hoz RE, Villamor JM, Mahíllo-Fernández I. Anxiety and depression predict musculoskeletal disorders in health care workers. Arch Environ Occup Health. 2017:72:39-44.

13. Wirth B, Humphreys BK, Peterson C. Importance of psychological factors for the recovery from a first episode of acute non-specific neck pain - a longitudinal observational study. Chiropr Man Therap. 2016;24:9. https://doi. org/10.1186/s12998-016-0090-2.

14. Ailliet $L$, Rubinstein SM, Knol D, van Tulder MW, de Vet HCW. Somatization is associated with worse outcome in a chiropractic patient population with neck pain and low back pain. Man Ther. 2016;21:170-6.

15. George SZ, Beneciuk JM. Psychological predictors of recovery from low back pain: a prospective study. BMC Musculoskelet Disord. 2015;16:49. https://doi. org/10.1186/s12891-015-0509-2.

16. Andersson GB. Epidemiological features of chronic low back pain. Lancet. 1999;354:581-5.

17. Pincus T, Burton AK, Vogel S, Field AP. A systematic review of psychological factors as predictors of chronicity/disability in prospective cohorts of low back pain. Spine. 2002;27:E109-20.

18. Hurwitz EL, Goldstein MS, Morgenstern H, Chiang L-M. The impact of psychosocial factors on neck pain and disability outcomes among primary care patients: results from the UCLA neck pain study. Disabil Rehabil. 2006;28:1319-29.

19. Hasenbring M, Hallner D, Klasen B. Psychological mechanisms in the transition from acute to chronic pain: over- or underrated? Schmerz. 2001:15:442-7.

20. Beneciuk JM, Bishop MD, Fritz JM, Robinson ME, Asal NR, Nisenzon AN, et al. The STarT back screening tool and individual psychological measures: evaluation of prognostic capabilities for low back pain clinical outcomes in outpatient physical therapy settings. Phys Ther. 2013;93:321-33

21. Industrial Insurance Medical Advisory Committee (last), Industrial Insurance Chiropractic Advisory Committee. Reducing disability: psychosocial determinants influencing recovery (PDIR): Washington State Department of Labor and Industries; 2016. [cited 2016 Sep 10]. Available from: http://www.Ini.wa.gov/ClaimsIns/Files/OMD/IICAC/ 2016PDIRResourceFinal.pdf

22. Ramond-Roquin A, Bouton C, Gobin-Tempereau A-S, Airagnes G, Richard I, Roquelaure $Y$, et al. Interventions focusing on psychosocial risk factors for patients with non-chronic low back pain in primary care--a systematic review. Fam Pract. 2014;31:379-88.

23. Engel GL. The need for a new medical model: a challenge for biomedicine. Science. 1977:196:129-36.

24. Gliedt JA, Schneider MJ, Evans MW, King J, Eubanks JE. The biopsychosocial model and chiropractic: a commentary with recommendations for the chiropractic profession. Chiropr Man Therap. 2017;25:16. https://doi.org/10. 1186/s12998-017-0147-x

25. Hill JC, Dunn KM, Lewis M, Mullis R, Main CJ, Foster NE. A primary care back pain screening tool: identifying patient subgroups for initial treatment. Arthritis Rheum. 2008;59:632-41.
26. Hay EM, Dunn KM, Hill JC, Lewis M, Mason EE, Konstantinou K, et al. A randomised clinical trial of subgrouping and targeted treatment for low back pain compared with best current care. The STarT Back Trial Study Protocol. BMC Musculoskelet Disord. 2008:9:58. https://doi.org/10.1186/ 1471-2474-9-58

27. Kongsted A, Andersen CH, Hansen MM, Hestbaek L. Prediction of outcome in patients with low Back pain--a prospective cohort study comparing clinicians' predictions with those of the Start Back tool. Man Ther. 2016;21:120-7.

28. Hill JC, Vohora K, Dunn KM, Main CJ, Hay EM. Comparing the STarT back screening tool's subgroup allocation of individual patients with that of independent clinical experts. Clin J Pain. 2010;26:783-7 doi.org/10.1097/AJP. 0b013e3181f18aac.

29. Foster NE, Mullis R, Hill JC, Lewis M, Whitehurst DGT, Doyle C, et al. Effect of stratified care for low Back pain in family practice (IMPaCT Back): a prospective population-based sequential comparison. Ann Fam Med. 2014;12:102-11.

30. Keele University-STarT Back Screening Tool. [cited 2016 Oct 20]. Available from: https://www.keele.ac.uk/sbst/startbacktool/

31. Field J, Newell D. Relationship between STarT Back screening tool and prognosis for low back pain patients receiving spinal manipulative therapy. Chiropr Man Therap. 2012;20:1-8.

32. Kongsted A, Johannesen E, Leboeuf-Yde C. Feasibility of the STarT back screening tool in chiropractic clinics: a cross-sectional study of patients with low back pain. Chiropr Man Therap. 2011;19:10. https://doi.org/10.1186/2045-709X-19-10.

33. Field J, Newell D. Clinical outcomes in a large cohort of musculoskeletal patients undergoing chiropractic Care in the United Kingdom: a comparison of self- and National Health Service-Referred Routes. J Manip Physiol Ther. 2016;39:54-62.

34. Newell D, Field J, Pollard D. Using the STarT Back tool: does timing of stratification matter? Man Ther. 2015:20:533-9.

35. Morso L, Kongsted A, Hestbaek L, Kent P. The prognostic ability of the STarT Back tool was affected by episode duration. Eur Spine J. 2016;25:936-44.

36. Irgens $P$, Lothe LR, Kvammen OC, Field J, Newell D. The psychometric profile of chiropractic patients in Norway and England: using and comparing the generic versions of the STarT Back 5-item screening tool and the Bournemouth questionnaire. Chiropr Man Therap. 2013;21:41. https:// doi.org/10.1186/2045-709X-21-41.

37. Gallup, Inc. Gallup-palmer College of Chiropractic Annual Report: Americans' perceptions of chiropractic. 2015. Available from: http:// www.palmer.edu/uploadedFiles/Pages/Alumni/gallup-report-palmercollege-2016.pdf

38. Kamper SJ, Apeldoorn AT, Chiarotto A, Smeets RJEM, Ostelo RWJG, Guzman J, et al. Multidisciplinary biopsychosocial rehabilitation for chronic low back pain. Cochrane Database Syst Rev. 2014;9:CD000963.

39. Stoltzfus JC. Logistic regression: a brief primer. Acad Emerg Med. 2011;18: 1099-104.

40. Khan $Y$. The STarT back tool in chiropractic practice: a narrative review. Chiropr Man Therap. 2017;25:11.

41. National Institute for Mental Health. NIMH » Any Anxiety Disorder. [cited 2018 Jun 29]. Available from: https://www.nimh.nih.gov/health/statistics/ any-anxiety-disorder.shtml\#part_155097

42. National Institute for Mental Health. NIMH » Major Depression. [cited 2018 Jun 29]. Available from: https://www.nimh.nih.gov/health/statistics/ major-depression.shtml

43. Robinson HS, Dagfinrud H. Reliability and screening ability of the StarT Back screening tool in patients with low back pain in physiotherapy practice, a cohort study. BMC Musculoskelet Disord. 2017;18:232. https://doi.org/10. 1186/s12891-017-1553-X

44. Oka H, Matsudaira K, Fujii T, Kikuchi N, Haga Y, Sawada T, et al. Estimated risk for chronic pain determined using the generic STarT Back 5-item screening tool. J Pain Res. 2017;10:461-7.

45. Thomas S, Jenkins R, Burch T, Calamos Nasir L, Fisher B, Giotaki G, et al. Promoting mental health and preventing mental illness in general practice. London J Prim Care (Abingdon). 2016;8:3-9.

46. Murphy DR, Hurwitz EL. Application of a diagnosis-based clinical decision guide in patients with low back pain. Chiropr Man Therap. 2011;19:26. https://doi.org/10.1186/2045-709X-19-26.

47. Murphy DR, Hurwitz EL. Application of a diagnosis-based clinical decision guide in patients with neck pain. Chiropr Man Therap. 2011;19:19. https:// doi.org/10.1186/2045-709X-19-19. 
48. Hill JC, Whitehurst DG, Lewis M, Bryan S, Dunn KM, Foster NE. Comparison of stratified primary care management for low back pain with current best practice (STarT Back): a randomised controlled trial. Lancet. 2011;378:1560-71 doi.org/10.1016/S0140-6736(11)60937-9.

49. Murphy DR. Clinical Reasoning in Spine Pain. Volume I: Primary Management of Low Back Disorders Using the CRISP Protocols, vol. 1; 2013.

Ready to submit your research? Choose BMC and benefit from:

- fast, convenient online submission

- thorough peer review by experienced researchers in your field

- rapid publication on acceptance

- support for research data, including large and complex data types

- gold Open Access which fosters wider collaboration and increased citations

- maximum visibility for your research: over $100 \mathrm{M}$ website views per year

At BMC, research is always in progress.

Learn more biomedcentral.com/submissions 